Article

\title{
Design of a Wearable Textile Broadband RF Ambient Power Harvester
}

\author{
Weihao Qiu ${ }^{1, *}$, Zhirun Hu ${ }^{1}$, Shimeng Wang2 and Liquan Xiao ${ }^{3}$ \\ 1 Department of EEE, School of Engineering, University of Manchester, Manchester, UK \\ 2 Department of EEE, School of Engineering, University of Manchester, Manchester, UK \\ 3 College of Computer, National University of Defense Technology, Changsha, Hunan, China; \\ xiaoliquan@nudt.edu.cn \\ * Correspondence: qiuweihao2015@gmail.com; Tel.: +86-186-1822-1899
}

\begin{abstract}
As the rapid development of the communication industry, especially the 5G communication and IOT, there will be plenty of electromagnetic (EM) waves in the free space, which carry lots of energies. However, some of these energies are wasted in free space. To reuse these wasted energies, significantly growing interests are towards to the energy harvesting antennas. This paper is aimed to investigate a wearable antenna which can transfer RF energy from ambient sources to direct current by a soft and portable textile antenna. Among the numerous signals in the mobile network, the GSM 1800, 3G, WiFi and 4G/5G will be chosen due to the city signal intensity distribution. Hence, a corresponding triple bands antenna has been designed to cover those frequency bands. The CST STUDIO SUITE is used in a whole process of antenna design and simulation. The proposed antenna is a hooked dipole antenna with tuning bar (HATB) whose ends have a small folded part in each side for better bandwidth performance. The presented antenna provides a wide operating band from $1.8 \mathrm{GHz}$ to $2.5 \mathrm{GHz}$ below $-10 \mathrm{~dB}$ in return loss excepting the sector from $2.08 \mathrm{GHz}$ to $2.39 \mathrm{GHz}$. And methods to overcome interference between antennas are found. Furthermore, using the corresponding rectifier to achieve the RF/DC conversion. The overall efficiency of the whole rectifier is about $56.8 \%$, and the output power level of the antenna system is $45.92 \mathrm{nW}$. The experimental results could indicate that my textile hooked antenna harvester is a good choice for the charging system of personal wearable attachment, which could achieve low power absorbing for long-distance at anytime and anywhere. And the wearable antenna could be used for active RFID tag antenna as well with the power harvesting antenna for power supply to cover the needs of various environments and applications.
\end{abstract}

Keywords: EM waves, harvester, wearable antenna, 5G, HATB, rectifier, efficiency, RFID tag

\section{Introduction}

Nowadays, energy conservation and emission reduction is an emerging and potential technology. An alternative method of new energy access is necessary so that ambient RF energy harvester is considered as abroder range of suitable power source for all kinds of applications. There are some new technologies about energy harvesting, for examples: piezoelectric, photovoltaic, and thermoelectric energy harvesting systems, which have even been used to power up low power devices [1]. However, these harvesting devices have some limitations, and they are vulnerable or environment polluted or limited by the time and distance. A key issue is the persistence of power: some only available during the daylight time.

Compared with these energy harvesting methods, a significantly growing interest has been towards the power harvesting antenna in recent years, which is wearable and could absorb energy without time and distance limitation. It is put into use in all kinds of fields, especially under the rapid development of 5G communication, such as IoT (Internet of Thing) and wearable medical devices [2], which is composed of internet, physical devices, vehicles and sensors [3]. In [4], Autonomous 
Wireless Sensors (AWSs) is proposed by duplicating the transceivers and also the power source using a hybrid storage system. Current AWS technology allows the development of many IoT-based applications in which the power source is a critical issue. Optimization of the system considers all factors obtaining an energy efficient AWS, paving the way towards autonomous sensors by adding an energy harvesting antenna which will influence the life span and realiability of the AWS. These energy harvesting function could realize by this article project. There are several body antennas which are applied to the professional field as the charging systems, such as in the sensor application of health care workers, firefighters, and the military. Besides, wearable antenna can also be used for the health monitoring of the elderly, young people and athletes [5].

Meanwhile, there are all kinds of antennas, such as monopole, dipole, loop, helical antenna, patch antenna and so on. Correspondingly, all kinds of power harvesting antennas were presented. Recently, RF ambient power harvester is growing to be a hot topic. In 2014, the UK Technology Strategies Board (TSB) confirmed there are 74 principal investigators, 130 companies and 29 UK universities studying in the field of energy harvesting. Such as the patch antennas are made of a metal printed patch, conductive ground plane and nonconductive fabric substrate [7], and the broad-band rectangular patch antenna with a pair of wide slits has a peak antenna gain about 7.2 dBi by Kin-Lu Wong in [8]. These antennas are compact and can cover a wide operating band. However, the performance of IL (insertion loss) and efficiency is not good, and most importantly, it is not flexible. Spiral antenna is analyzed in the presence of a conducting plane reflector in [9-10] by H. Nakano, S. Arai and etc., the spiral antenna also uses metal-printing textile fabric, which has smaller size than monopole antenna and has wider band range from 1.2 to $5 \mathrm{GHz}$ in 9, and the total measured RF-DC conversion efficiency is maintained above $30 \%$ across the entire frequency range with a peak value of $61 \%$ achieved at $1.2 \mathrm{GHz}$. But the radiation pattern and gain is limited, and it has asymmetry antenna gain and its polarization mode is elliptical polarization. In [11], the improvement of RF Wireless Power Transmission (WPT) is presented by using a circularly polarized retrodirective antenna array with EBG structures at $5.2 \mathrm{GHz}$. Owing to the mutual coupling reduction, at the broadside direction, improvements of approximately $11.32 \%$ and $12.45 \%$ and gain enhancement of 0.4-0.8 dB were realized using $5 \times 5$ and $10 \times 10 \mathrm{CP}$ RDAs with EBGs compared without the EBGs. Furthermore, it has a narrow $-10 \mathrm{~dB}$ reflection bandwith ranging from $5.12-5.30 \mathrm{GHz}$. Graphene is a new type of nanomaterial with the thinnest, strongest conductivity and thermal transmission. The graphene antenna was tried many times in our experiment, lithography was done with photoresist. It is so fragile and vulnerable and always been destroyed under the design and operation.

Hence, the light wearable antenna was explored. Recently, textile wearable antennas have developed quickly with the rapid development of the manufacturing technologies of conductive fiber, which have attracted more and more researchers to study the new kind of material to create new smart antennas. Due to the flexibility and comfort of antenna textile, and lightweight of the substrate textile, the wearable antenna is a good choice for body wearing. Using the flexible conductive textiles, which can seamlessly weave the wearable antenna into clothes. Moreover, for the body wearable antenna, the good induced EM field and radiation pattern of antenna is essential to the power-efficient devices $[12,13]$. When the harvester is put into use in small sensors, this can extend the sensors life and people do not need to change the dead battery frequently [14,15]; and even in the health care system such as the eyes lenses which is used for eyes pressure measuring [16]. In [17], a printed wearable dual-band antenna is presented operating at ultra-high frequency antenna 
(2.5 GHz/4.5 GHz) for WPT applications. The antennas were based on split ring resonator. The shape of the antenna provides multiple dimensions that can be optimized for the desired frequency. It is near omni-directional radiation pattern and the transmission efficiency was 3.94\% for the first resonance frequency and $2.29 \%$ for the second one which does not vary significantly due to change of distance. Besides, the wearable broadband folded dipole rectenna for FM reception was invented by the Jung-Sim Roh, and etc., this rectenna provided a wide operating low-frequency band of 80.5 $\mathrm{MHz}$ to $130 \mathrm{MHz}$ at $5 \mathrm{~dB}$ reflection coefficient regardless of the textile bending [18]. However, in the past research, the RF power harvester was mainly focused on the single or dual-band RF antenna [19, 20]. In this project, the multiband RF ambient power harvester is designed and fabricated, the frequency of sources ranges from $1.8 \mathrm{GHz}$ to $2.5 \mathrm{GHz}$, which include GSM 1800, 3G, WiFi, 4G and even $5 \mathrm{G}$ signal. The $5 \mathrm{G}$ will be commercial soon, and with the coverage of $5 \mathrm{G}$ base station, which has wider bandwidth and stronger signal power density due to beamforming and good spectral efficiency owing to the MIMO technology, the $5 \mathrm{G}$ will accelerate the development of harvesting antenna. This wideband antenna will be able to absorb 5G signal which will be covered worldwide in the future. And compared with past charging equipment, this wireless charging antenna could receive the energy above 10 meters range anytime and anywhere, which is suitable for long-distance and low power equipment charging, such as the motion detecting, body function monitoring and emergency services and etc. Hence, I choose this dipole antenna which has wide bandwidth, good gain and omni-directional radiation pattern, and above all, it is flexible, comfortable and bended as will which could be sewed in the rescure-workers suits for charging the active RFID tag, in the fire and earthquake disaster relief, it is easier and more efficient to accurately find the location of people without the environment and distance limitation. In this condition, the green RF-WPT architecture is presented by this havester, and a promising network will come true in the future in which smart devices can be recharged by these green resources.

Besides, the antenna impedance-matching is needed, which can nearly realize no return loss in the process of transmission and would get entire energy from received sources ideally. Then, the permittivity of wearable fabric is measured by the waveguide testbed. Moreover, using software optimization, the antenna has been tuned to get the best possible performance. After this, the wearable antenna is sewed on the textile fabric. In the end, the wearable textile broadband hooked antenna harvester is gotten by connecting to the rectifier which could achieve the RF-DC conversion. The overall efficiency of whole rectifier is assumed about $70 \%$ in the specification. In this paper, my task plan is that a multi-band flexible antenna with good efficiency, comfortable wearing and simple can be used for charging power devices or store the energy in batteries [18,19]. In [21-23], various wearable antennas were discussed under bending and on-body conditions. The wearable antenna has some frequency shift when on different conditions, especially on body, but the bandwidth has no much change. The rest of the paper is organized as follows. The main characteristics of the wearable antenna and rectifier are introduced in section 2 . Section 3 presents the antenna simulation methods and results. In section 4, the full prototype of harvester is fabricated and measured. Finally, the conclusions are drawn in section 5 . 


\section{Background Materials and Characterization}

\subsection{Mobile network frequency band}

At present, the mobile network frequency ranges from $0.8 \mathrm{GHz}$ to $4 \mathrm{GHz}$, which include GSM 900, GSM 1800, 3G, 4G, WIFI and the coming 5G signal as table 1 shown below. The frequency band of GSM 900 is $880 \mathrm{MHz}$ to $960 \mathrm{MHz}$ in the UK, the frequency band of GSM 1800 is $1710 \mathrm{MHz}$ to 1880 MHz, the 3G signal is UMTS 2100 has the frequency band which ranges from $1920 \mathrm{MHz}$ to $2170 \mathrm{MHz}$. The $4 \mathrm{G}$ is LTE $\mathrm{B} 42$ has the frequency at $3.4-3.6 \mathrm{GHz}$ which has the same frequency distribution as the 5G NR n41 band, so if the 5G base station is built, the antenna could support the 5G signal absorbing as well. And different countries and operators have different band allocation in same mobile network type, this wide band could cover many changes.

Table 1. Mobile RF band distribution and power density in the UK.

\begin{tabular}{cccccc}
\hline Mobile signal & 2G & 2G & 3G & WiFi & 4G/5G \\
\hline $\begin{array}{c}\text { Band } \\
\text { Frequencies } \\
\quad \text { GSM 900 }\end{array}$ & GSM 1800 & UMTS 2100 & WiFi & LTE B41/NR n41 \\
$\begin{array}{c}\text { Average S } S_{\text {av }} \\
(\mathrm{nW} / \mathrm{cm})\end{array}$ & $0.88-0.96$ & $1.71-1.88$ & $1.92-2.17$ & $2.4-2.483$ & $2.496-2.69$ \\
$\begin{array}{c}\text { Maximum Sav } \\
\left(\mathrm{nW} / \mathrm{cm}^{2}\right)\end{array}$ & 39 & 0.5 & 0.46 & 0.38 & 0.35 \\
\hline
\end{tabular}

The power density $\mathrm{S}_{\mathrm{av}}(\mathrm{nW} / \mathrm{cm} 2)$ is also an important point which needs to be considered for frequency choosing. In order to know the input power density in the UK environment, a citywide RF spectral survey was cited which is about the power density within the ultrahigh-frequency (0.3-4 $\mathrm{GHz}$ ) part of the frequency spectrum. As shown in table 1, the maximum power density of 3G and wifi are larger than other signal sources.

To decide which band to choose, the Friis Equation needs to be known first. There are two antennas are lined up for signal transmitting. The receiving antenna (with gain $G_{r}$ ) receives the signal from another antenna (with gain $G_{t}$ ), with a distance $\mathrm{R}$, and operating at frequency for wavelength lambda. Assuming that both antennas are matched to the generator and load, and two antennas are in the free space region which means there is no obstructions nearby.

For the moment, if the transmitting antenna is omnidirectional, lossless, and the receiving antenna is placed in the far-field region of the transmitting antenna, the resulting received power can be written as:

$$
P_{r}=\frac{P_{\max }}{4 \pi r^{2}} G_{t} \frac{\lambda^{2}}{4 \pi} G_{r}=\left(\frac{\lambda}{4 \pi r}\right)^{2} P_{\max } G_{t} G_{r} .
$$

This is called Rader Range Equation, which is also known as the Friis Transmission Formula. It relates the free space path loss, antenna gains and wavelength to the received and transmitting powers.

Another useful form of the Friis Transmission Equation is changed to a form, as seen in [24]. Since wavelength is equal to frequency $f$ over the speed of light $c$, we have the Friis Transmission Formula in terms of frequency: 


$$
P_{r}=\left(\frac{c}{4 \pi r f}\right)^{2} P_{\max } G_{t} G_{r} .
$$

From above equation, we know that the higher the frequency is, the lower receiving power it will have. Therefore, the 5G FR2 Millimeter-wave is not considered into use, we choose these lower frequency bands.

$$
\lambda={ }^{c} / f
$$

From the above equation, the lower the signal frequency is, the longer the wavelength will be. And the length of dipole which is $\lambda / 2$ will be longer as well, so it will be too long to build the antenna. And if we choose the GSM 900, this signal will interfere with 3G and WiFi signal and cannot get a good performance in S11 and efficiency. And the rectifier that we use cannot deal with the wide frequency band. Therefore, the frequency of $900 \mathrm{MHz}$ which is far away from the other frequencies should be abandoned. In order to support more bands, a single wideband antenna which could harvest WIFI, 4G and 5G simultaneous was designed, we could alternate the harvesting band according the actual demand.

From the above analysis, finally, I choose the GSM 1800, UMTS 2100, WiFi and 4G/5G mobile network signal as the harvesting sources.

\subsection{Wearable antenna}

The silver high conductive yarns we used were from STATEX Ltd which was was a hybrid yarns containing metal fiber. The conductivity is an essential parameter of conductive material; it represents the ability to conduct electricity. The conductivity of this silver yarns is $2.09^{*} 105 \mathrm{~S} / \mathrm{m}$. Meanwhile this silver yarn is flexible and folding that could fulfill various environment and application needs, which is not like the functionally limited coated yarns with fragile and unbended performance. The antenna using this silver high conductive yarns allow higher geometrical accuracies of the frame of the antenna and could provides multiple dimensions that can be optimized for the desired frequency [25].

As we know, different antennas with different properties, so that the kind of antenna need to be decided according to actual needs. Generally, the working efficiency of the antenna is related to the volume of the antenna. At the same time, the size of the antenna is also associated with the wavelength. I choose the half-wave dipole antenna as the harvesting antenna, which has omnidirectional radiation pattern and broader bandwidth, at the same time, it can be designed by sewing with fabric efficiently and it is comfortable and flexible for body wearing, those fulfill all requirements. It is formed of 2 symmetry quarter wavelength elements which are two identical conductive elements such as wires or rods. And the dipole is fed in the center where the impedance reaches to its lowest value. The impedance in each end rises to highest value which reaches to infinity because the open circuit, where the current is zero, but the voltage reaches to maximum value.

Moreover, for the purpose of having a good understanding about antenna performance, antenna parameters are necessary to discuss:

- Gain and efficiency. The gain is relative to the antenna's directivity and electrical efficiency. Directivity describes the factor of power density enhancement in the maximum radiation direction with respect to the radiation of an isotropic. The gain of a receiving antenna is a parameter that is defined with respect to the antenna's ability of radio waves convert into input power. Thus, the harvester will get more input power with the high gain. 
- Resonant frequency. The resonant frequency is decided by the size of the antenna. But in practical, the size of antenna is longer than the theoretical value. The antenna's capacitance and inductance are decided by its size and the substrate material. It is proved that the larger the antenna size and the value of material's permittivity are, the lower the resonant frequency becomes.

- Impedance Bandwidth. The range that can operate efficiently over one value, such as $-10 \mathrm{~dB}$ in $\mathrm{S} 11$, is the desired bandwidth of the antenna. S11 is the reflection coefficient, which represents the ratio of the complex value of the reflected wave to that of the incident wave. Outside this value, the reactance will rise to a level that may be too high for good performance. The wider bandwidth it is, we will harvest more energy, and we can avoid the errors which are resulted by the resonant frequency shifting.

- To increase the bandwidth of an antenna, a lot of methods can be taken. One of which is the use of thicker conductors. Another way is the variant type of antenna. For example, a folded dipole which is described fully in Chapter 3 has a wider bandwidth than a non-folded one. In fact, looking at a standard television antenna, it is possible to see both of these features included.

- Radiation pattern. It is depends on the shape of antenna and changed with frequency. Most antennas show a pattern of "lobes" or maxima of radiation. The main lobe is the most significant strength of the radio waves, which is the desired direction of signal receiving. Particularly, the front to back lobe ratio will decrease rapidly if the antenna is operated outside a given bandwidth.

\subsection{Substrate material}

The property of substrate material affects the antenna resonant frequency (fr), bandwidth and S11 (reflected constant). The substrate uses the nonconductive textile material, such as polyamide, silk, fleece and cotton. The fabric I used is the polyamide which is comprised of $50 \%$ meta-aramid and $50 \%$ FR lensing fiber.

The material property is about the permittivity $(\varepsilon)$ and loss tangent. When a medium is in the electric field, it will produce induced charge and weaken the electric field. Permittivity is a measurement of how an electric field affects and is affected by a medium. The permittivity describes how much unit charge in the electric field, which is generated in that medium. We know that the permittivity is:

$$
\varepsilon=\varepsilon_{\mathrm{r}} \varepsilon_{0}
$$

where $\varepsilon_{\mathrm{r}}$ is the effective permittivity of the material, and $\varepsilon 0=8.8541878176 . . \times 10-12 \mathrm{~F} / \mathrm{m}$ is the vacuum permittivity. As is known to all,

$$
\lambda=v / f=\frac{c}{\sqrt{\varepsilon} * f}
$$

The $\mathrm{c}$ is the speed of light, and $\mathrm{f}$ is the frequency of a mobile network signal. The $\varepsilon$ represents the permittivity of substrate which is the parameter needs to be measured. From equation 5 , the permittivity will influence the antenna size. 


\subsection{Rectifier}

To get the DC source, a rectifier is chosen which can convert the RF signal to DC. And due to the input RF power from ambient sources is multi-tone, the power fluctuates with the frequency ranging, and the output impedance of the antenna changes over time. A rectifier is required for solving these unstable problems. Furthermore, before charging the DC energy into a storage element, a capacitor is needed, making the pulsating dc voltage after rectification becomes a relatively stable dc voltage and beneficial to the battery or the load stress avoidance [26]. The harvester constantly charges the storage batteries or the load device which is automatically loaded by the storage capacitor when the capacitor voltage reaches an upper limit. Sometimes, the obtained DC voltage is a few hundred millivolts, which usually cannot satisfy the automatic activation of dc-dc converters. To overcome this shortcoming of battery, auxiliary converter could be considered to the converter start-up phase [27]. And the antenna array can be used to enhance the output voltage as well.

\section{Methods and results of antenna simulation}

\subsection{Antenna design}

\subsubsection{CST software analysis and impedance matching}

It is known that this project is operated on CST to define the structure, material properties and discrete signal port of the folded received dipole antenna. Then we can get simulated S-parameter, impedance, bandwidth, radiation pattern and gain of antenna. We also can observe the far-field, Efield and H-field of antenna. By doing this, we can get the proper and demanded antenna. The antennas need to be designed to be as omnidirectional as possible, while covering as much ambient RF source as possible. The properties of CST need to be adjusted to realize the optimized results. In numerical analysis, adaptive mesh refinement, or AMR needs to be done. This is a method of adapting the accuracy of a solution within certain sensitive or turbulent regions of simulation, during the solution is calculated.

As discussed above, the antenna can be viewed as a passive network of RLC, and the network will produce resonant impedance values. It is necessary to maximize the efficiency of energy-transfer by matching the port impedance of the RF antenna to the load in simulation. The port impedance of the antenna results from several factors including the size and shape of the RF antenna, the frequency of operation and its environment. The impedance usually is complex which is consisted of resistive elements as well as reactive ones. Mutual coupling decreases not just the antenna efficiency, but also it can alter the antenna's radiation pattern as well. Hence, many ways were tried to decrease the interference, such as remove the half part of loop antenna, fold the antenna, add small bar beside the element and even some nails between the element.

\subsubsection{Initial design of folded antenna}

The fabric is shown in figure 1a, firstly, the permittivity of the substrate needs to be measured for ensuring the antenna length. The waveguide WG 10 operating in TE10n mode which ranges from $2.5 \mathrm{GHz}$ to $3.5 \mathrm{GHz}$ is considered as the measuring instrument. The waveguide cell is formed by four boundaries cell which is used to clamp the measured fabric which is between two Perspex windows 
as shown in the figure $1 \mathrm{~b}$. This method forms a reflection transmission at interface boundaries and gets the Musil and Zacek expressions for transmission and reflection coefficients from example for four boundaries between microwave windows, which is proposed by the Musil and Zacek in 1986 in their text [28]. The reflection transmission expressions for the four boundaries problem are both complex variables and are solved by using the Newton-Raphson approximation [29,30].

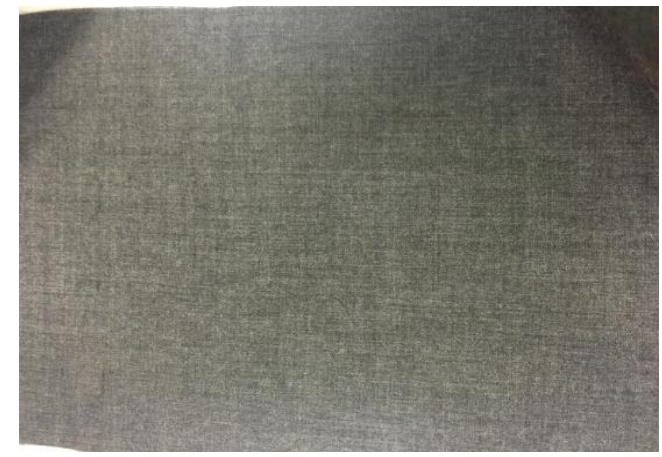

(a)

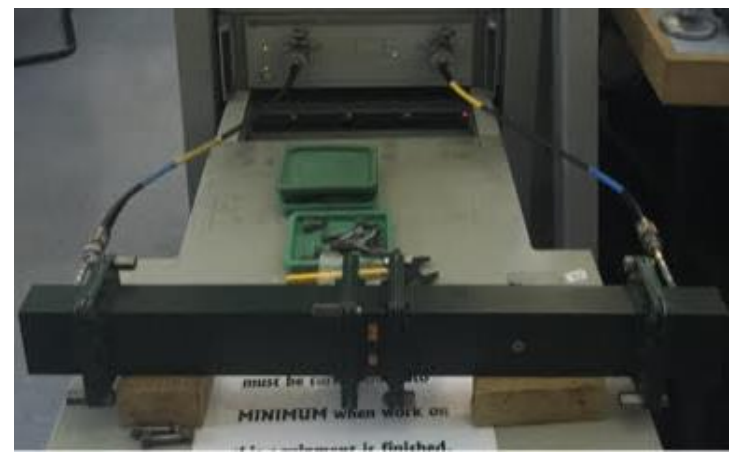

(b)

Figure 1. Substrate measuring environment, (a) test sample of the fabric substrate, (b) test instrument of waveguide WG 10 with two Perspex windows internally.

The measuring equipment is the 8510 Vector Network Analyser (VNA). Making an assessment of calibration accuracy by checking S11 and S22 using waveguide short circuit and checking phase using phase offset. Then we can obtain the real and imaginary permittivity results at each measuring frequency by using the Newton Raphson algorithm in the MATLAB. From the results, the permittivity is about $2.2 \mathrm{~F} / \mathrm{m}$ for simulation when the frequency is from $1.8 \mathrm{G}$ to $2.4 \mathrm{G}$. After knowing the estimated permittivity of substrate, we can confirm the single antenna size.

The length of the folded dipole antenna in frequency $f_{1}=1.8 \mathrm{GHz}$ is:

$$
l_{1}=\frac{1}{2} \lambda_{1}=\frac{1}{2} \frac{c}{\sqrt{\varepsilon} \times f_{1}}=\frac{3 \times 10^{8}}{2 \times \sqrt{2.2} \times 1.8 \times 10^{9}}=0.0562 \mathrm{~m}=56.2 \mathrm{~mm} .
$$

In the same way, the length of the folded dipole antenna in frequency $f_{2}=2.1 \mathrm{GHz}$ is $48.2 \mathrm{~mm}$; the length of folded dipole antenna in frequency $f_{3}=2.4 \mathrm{GHz}$ is $41 \mathrm{~mm}$.

The single folded antenna of each frequency needs to be designed separately. We know that the longer the length of antenna is, the lower the centered frequency will be. Using the CST to simulate the antenna performance. Generally, the dielectric is changed by the frequency, temperature, and surface roughness, the moisture content purity and homogeneity of the material [31]. Hence, the real size is not equal to the calculated values; it will fluctuate with the circumstance.

Firstly, the three chosen square loop antennas work not good in return loss and bandwidth, then many times optimisms are done to change the parameters of antenna, but it does not work properly as well. We know that the input impedance is different with different frequencies. It is hard to match the impedance of every frequency, especially the impedance of $1.8 \mathrm{GHz}$ and $2.1 \mathrm{GHz}$. And it cannot reach the good performance no matter how I adjust the parameters variable values. Hence, I try a new kind of antenna to improve the performance of the antenna.

I try to remove the top metal robs of antennas, then get this single hook-shaped antenna as shown in figure 2a, the hook antenna works at $1.8 \mathrm{GHz}$, of which length is $56.2 \mathrm{~mm}$ and width is 8 $\mathrm{mm}$. Set the port impedance $50 \mathrm{ohm}$. The $\mathrm{S} 11 \mathrm{in} 1.8 \mathrm{GHz}$ is $-23.538 \mathrm{~dB}$, bandwidth in $-10 \mathrm{~dB}$ RL is 180 $\mathrm{MHz}$. 


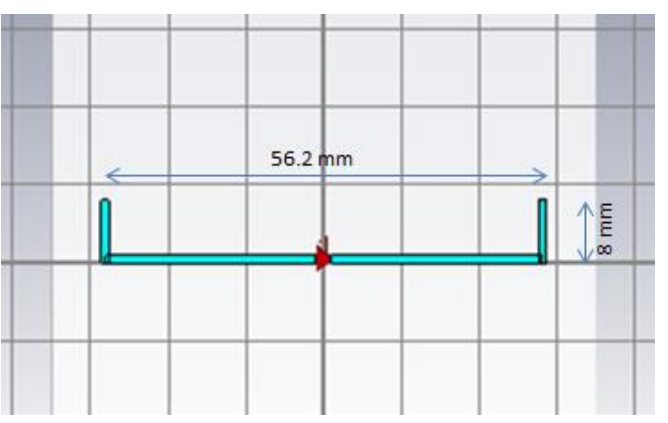

(a)

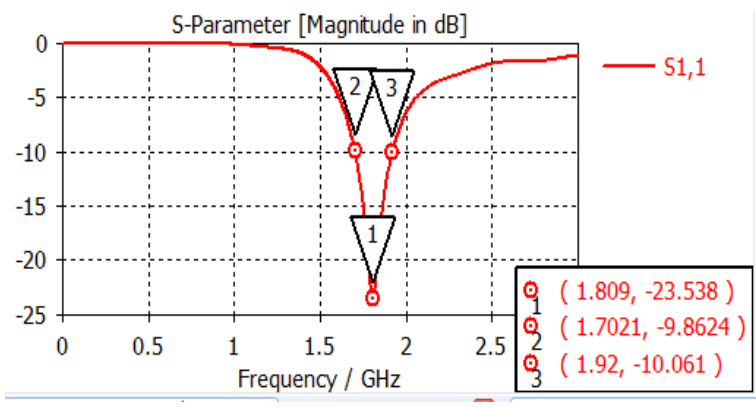

(b)

Figure 2. (a) The layout of the single hook antenna works at $f_{r}=1.8 \mathrm{GHz}$, (b) The reflected coefficient of the single hook antenna works at $\mathrm{f}_{\mathrm{r}}=1.8 \mathrm{GHz}$.

\subsubsection{Triple band hooked folded antenna}

The other two frequency antennas were simulated as well, and the antenna length was gotten. In order to avoid coupling and interference between two antennas, some nails were added in the mid of two antennas initially. This hook antenna is less influenced by the interference than the square loop dipole. Lastly, the triple band hooked antenna is designed. The layout of antenna is demonstrated in figure 3a, three antennas are all hooked shape, and are fed by one port. The impedance is $50 \mathrm{Ohm}$ can match the port impedance nicely. The length of antenna resonates at 1.8 $\mathrm{GHz}$ is $58 \mathrm{~mm}$ and the width is $8 \mathrm{~mm}$; when $\mathrm{fr}_{\mathrm{r}}=2.1 \mathrm{GHz}$, the length of antenna is $50 \mathrm{~mm}$ and the width is $5 \mathrm{~mm}$; the bottom antenna is transformed to a hook antenna whose length is $40 \mathrm{~mm}$ and width is $5 \mathrm{~mm}$. The reflected coefficient $\left(S_{11}\right)$ is demonstrated in figure $3 \mathrm{~b}$; it resonates at three frequency point. At $\mathrm{f}_{\mathrm{r}}=1.8 \mathrm{GHz}, \mathrm{S}_{11}=-26.032 \mathrm{~dB}$; at $\mathrm{f}_{\mathrm{r}}=2.1 \mathrm{GHz}, \mathrm{S}_{11}=-32.499$; at $\mathrm{f}_{\mathrm{r}}=2.47 \mathrm{GHz}, \mathrm{S}_{11}=$ $20.68 \mathrm{~dB}$. Nevertheless, the bandwidth of each resonant frequency is small, especially at $\mathrm{f}_{\mathrm{r}}=2.1 \mathrm{GHz}$ and $\mathrm{f}_{\mathrm{r}}=2.45 \mathrm{GHz}$.

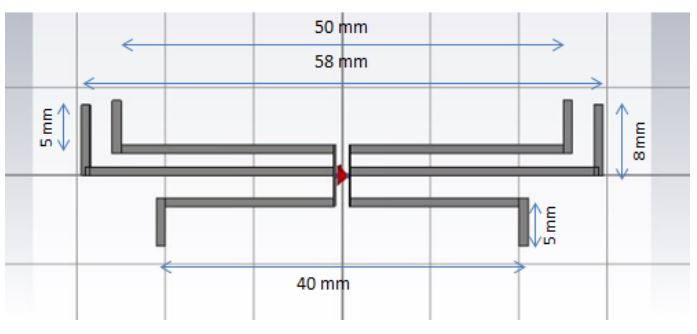

(a)

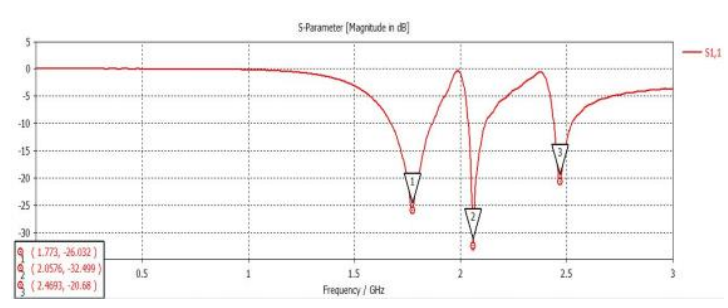

(b)

Figure 3. (a) The layout of the triple band hooked shape antenna, (b) The reflection coefficient of triple band hook shape antenna.

\subsubsection{The hook shape broadband antenna with tuning bar}

There are many ways to increase the antenna bandwidth. Such as, decrease the $Q$ factor, use the inter coupling, add harmonic oscillator or tuning bars, and make a slot in patch antenna. Due to the dipole antenna, two bending shape open circuit turning bar was added on up and downsides, which forms an asymmetry dipole antenna, it is exhibited in figure 4a.

The tuning bar can increase the impedance bandwidth of an antenna. Moreover, it can be put at a proper location of arm to adjust the impedance of antenna to get a better bandwidth in $-10 \mathrm{~dB}$. As 
shown in figure $4 \mathrm{~b}$, the $-10 \mathrm{~dB}$ bandwidth at each frequency are wider than before as well. At the point of $f_{r}=1.8 \mathrm{GHz}$, the bandwidth is: $f_{2}-f_{1}=1.92-1.67 \mathrm{GHz}=0.25 \mathrm{GHz}$; the second bandwidth is: $\mathrm{f}_{4}-\mathrm{f}_{3}=$ $2.2-2.04 \mathrm{GHz}=0.16 \mathrm{GHz}$; the third band width is: $\mathrm{f}_{6}-\mathrm{f}_{5}=2.61-2.41 \mathrm{GHz}=0.2 \mathrm{GHz}$.

The performance of $S_{11}$ bandwidth is excellent and can harvest three needed kinds of RF signal perfectly. Meanwhile, $-5 \mathrm{~dB}$ bandwidth of hook shape antenna is about $1.3 \mathrm{GHz}$ which is from 1.6 $\mathrm{GHz}$ to $2.9 \mathrm{GHz}$ except a small band in $2.3 \mathrm{GHz}$. Hence, this can be called wideband antenna in $-5 \mathrm{~dB}$.

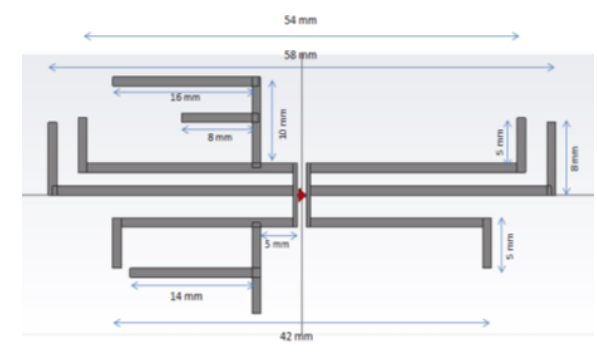

(a)

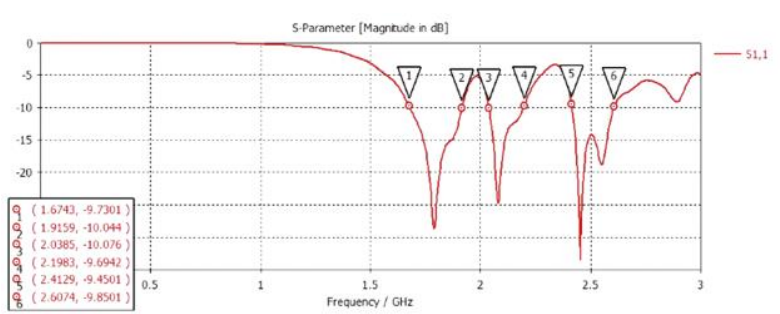

(b)

Figure 4. (a) The layout of hook shape broadband antenna with tuning bar, (b) Reflection coefficient of hook shape broadband antenna with tuning bar.

The surface current distribution is wider than the hook shape antenna without the tuning bar as well. There is some strong current distribution in the top tuning bar at $\mathrm{f}=2.1 \mathrm{GHz}$, this contributes to wider bandwidth of antenna at this frequency band. And the current distribution of bottom tuning bar is strong as well; this even can be viewed as forming a new band close to the $2.45 \mathrm{GHz}$ frequency band which can harvest the 4G/5G signal energies. All about this formed a broadband antenna.

\section{Harvester fabrication and measurement}

\subsection{Antenna fabrication}

After getting the simulated model of antenna, and with the help of technician from school of material, the wearable antenna which is sewed by the metal yarn in the fabric substrate is finished. Next, to use a special conductive adhesive to bond the textile antenna with the conductive wire which is deemed as the feed point, then, connects it to the $50 \mathrm{Ohm}$ coaxial cable. The coaxial cable is a type of special cable that has an inner conductor surrounded by a dielectric insulating layer, which is parceled by a tubular metallic shield and surrounded by a plastic jacket. It is used as a transmission line for radio frequency signals. After that, screw coaxial cable into the port of measuring instrument. The broadband hooked antenna with tuning bar (HATB) was sewed in the fabric substrate by using the silver yarn as shown in figure $5 \mathrm{a}$. The $\mathrm{S}_{11}$ measured by the Microwave Analyser is good and has a broadband bandwidth in $-10 \mathrm{~dB}$. As shown in figure $5 \mathrm{~b}$, the figure illustrates the $-10 \mathrm{~dB}$ operating band ranges from $1.64 \mathrm{GHz}$ to $2.08 \mathrm{GHz}$ and $2.4 \mathrm{GHz}$ to $2.59 \mathrm{GHz}$, where the simulated result is from $1.67 \mathrm{GHz}$ to $1.91 \mathrm{GHz}, 2 \mathrm{GHz}$ to $2.2 \mathrm{GHz}$ and $2.41 \mathrm{GHz}$ to $2.6 \mathrm{GHz}$. The measured result is similar with the simulated result in bandwidth. It is seen that the measured $S_{11}$ was about to combined as one broadband in $2 \mathrm{G}$ and $3 \mathrm{G}$ band, which is mainly caused by that the real fabrication could not ensure the accuracy of the antenna length, hence with the bandwidth increasing, and the resonance point of these two dipoles are so close that the two closed waves were incorporated as one broadband $S_{11}$ wave, moreover the irregular free space signal and difference between the simulated and real 
antennas also influence the results and lead to the wave change, this is reasonable in the practical making. And as the textile antennas are porous materials and the presence of air approaches the relative permittivity to reduce, the low dielectric constant reduces the surface wave losses that are tied to guided wave propagation within the substrate. Therefore, lowering the dielectric constant increases spatial waves and hence increases the impedance bandwidth of the antenna [25].

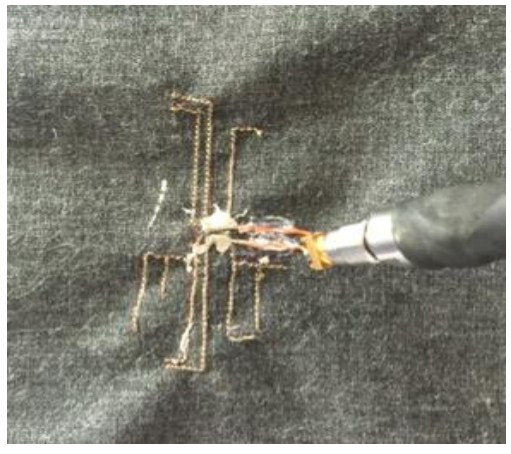

(a)

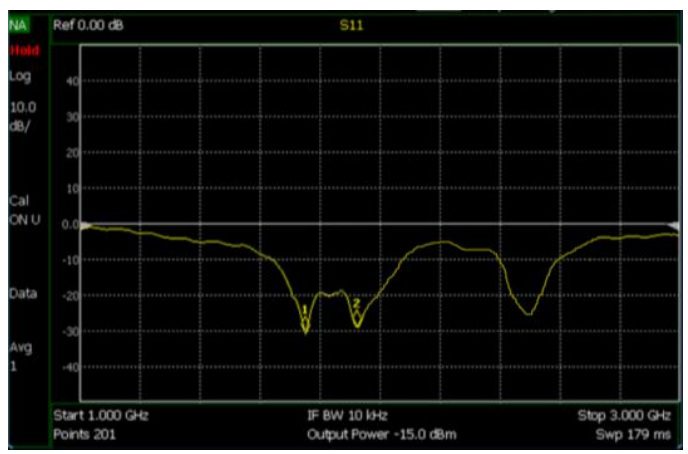

(b)

Figure 5. (a) Proto of broadband HATB in the fabric substrate, (b) Measured reflection coefficient of the fabricated broadband HATB in the fabric substrate.

\subsection{Radiation pattern measurement}

The simulated radiation pattern is represented in the far-field monitor which can be viewed as $3 \mathrm{D}$, polar and 2D mode. The antenna simulated radiation pattern at three operating frequency bands is shown as below. It includes a 3D simulation pattern and polarization pattern. As shown in figure 6 , the $3 \mathrm{D}$ simulated radiation pattern at $1.8 \mathrm{GHz}$, the gain is about $1.927 \mathrm{~dB}$, and the radiation efficiency is $-0.312 \mathrm{~dB}(93.1 \%)$. It is an omnidirectional pattern and looks like a doughnut. At $2.1 \mathrm{GHz}$, the gain is $1.864 \mathrm{~dB}$ and the radiation efficiency is $-0.52 \mathrm{~dB}(88.7 \%)$. At $2.45 \mathrm{GHz}$, the gain is $2.236 \mathrm{~dB}$ and the radiation efficiency is $-0.37 \mathrm{~dB}(91.8 \%)$.

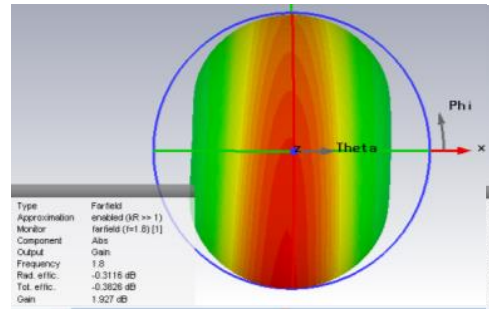

(a)

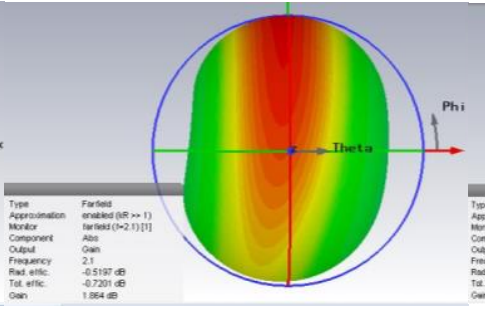

(b)

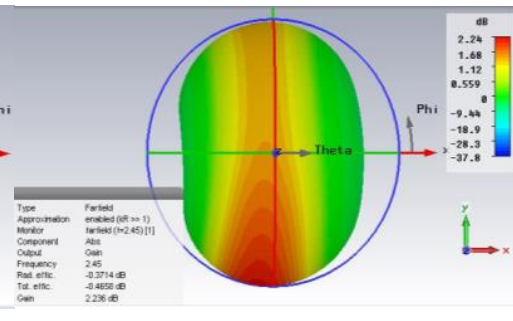

(c)

Figure 6. 3D simulated radiation pattern of $\mathrm{HTAB}$ at: $(\mathbf{a}) \mathrm{f}=1.8 \mathrm{GHz},(\mathbf{b}) \mathrm{f}=2.1 \mathrm{GHz}, \mathbf{c}) \mathrm{f}=2.45 \mathrm{GHz}$.

The simulated polar radiation pattern in the $\mathrm{E}$ plane or $\mathrm{H}$ plane is formed by a cut angle of theta $(\theta)$ or phi $(\varphi)$. The E-plane is formed when the $\theta=90^{\circ}$ (XY plane), it is like a transverse number 8 shape and it is the radiation pattern about electrical field in three different operating frequencies, as presented in following figures. The distribution of electrical field is related to the surface current distribution, which is mainly focused on the mid part of antenna. The maximum gain is $1.93 \mathrm{~dB}$ when the $\varphi=266^{\circ}$ which is located in the bottom part of antenna at $1.8 \mathrm{GHz}$, and angular width of the half- 
power beamwidth is $82.4^{\circ}$. And when the frequency is $2.1 \mathrm{GHz}$, the maximum gain is $1.87 \mathrm{~dB}$ at the $\varphi=93^{\circ}$ which is located in top part of antenna. The angular width of $3 \mathrm{~dB}$ is $78.3^{\circ}$. At same time, at $2.45 \mathrm{GHz}$, the gain of main lobe is $2.25 \mathrm{~dB}$ at $\varphi=263^{\circ}$, which is located in the bottom of antenna, the $3 \mathrm{~dB}$ angular width is $75.4^{\circ}$.

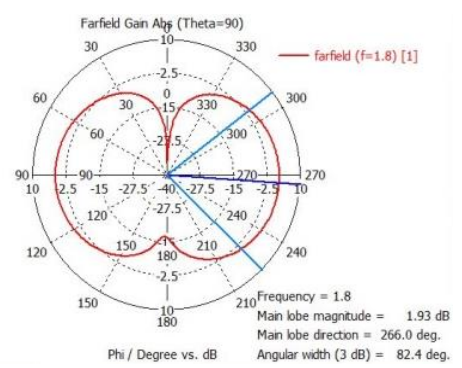

(a)

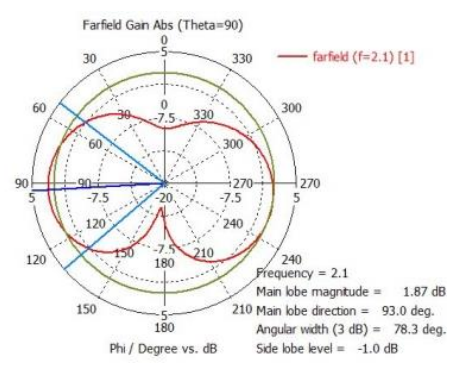

(b)

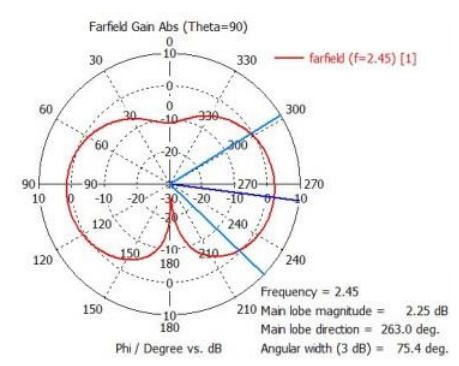

(c)

Figure 7. The E-plane radiation pattern of hooked wideband antenna at: $(\mathbf{a}) \mathrm{f}=1.8 \mathrm{GHz},(\mathbf{b}) \mathrm{f}=2.1 \mathrm{GHz}$, (c) $\mathrm{f}=2.45 \mathrm{GHz}$.

The $\mathrm{H}$ planes are all circle shape and omnidirectional in different frequencies, which are formed when the cut angle $\theta=0^{\circ}(\mathrm{YZ}$ plane), the gain of $\mathrm{H}$ plane in all direction is almost same. At $1.8 \mathrm{GHz}$, the simulated radiation pattern of $\mathrm{H}$ plane is a circle and the abs gain is $1.7 \mathrm{~dB}$; at $2.1 \mathrm{GHz}$, the abs gain $1.58 \mathrm{~dB}$; at $2.45 \mathrm{GHz}$, the main lobe gain is $1.47 \mathrm{~dB}$.

The radiation pattern is measured in an anechoic chamber as seen in figure 8 . The anechoic wall of this chamber will harvest unwanted signal. The wearable antenna is flexible; hence I stabilize the antenna on a hard board and then put it on a foam. Before the measurement, the calibration and loss measurement needs to be done for accurate measurement by using the horn antenna.

When measuring the E-plane, the antenna was put at horizontal plane, as shown in figure 8a. When measuring the H-plane, the antenna needs to be put at vertical plane, as seen in figure $8 \mathrm{~b}$. Another side is a standard dipole transmitter antenna, and the measured antenna should be parallel with this dipole. The distance of two antennas is 1.75 meters, as shown in figure 8c. The principle of the pattern measurement is based on the gain measurement on the received antenna under-tested in another transmitted antenna which operates on same bandwidth with the measured antenna.

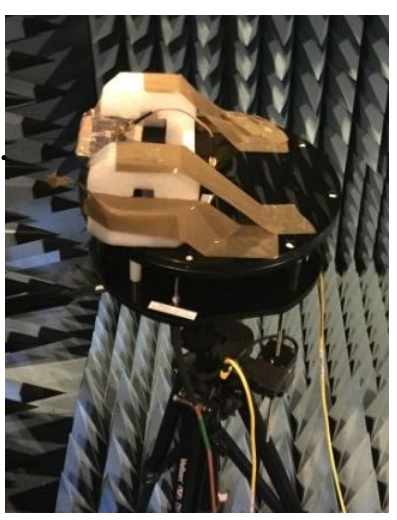

(a)

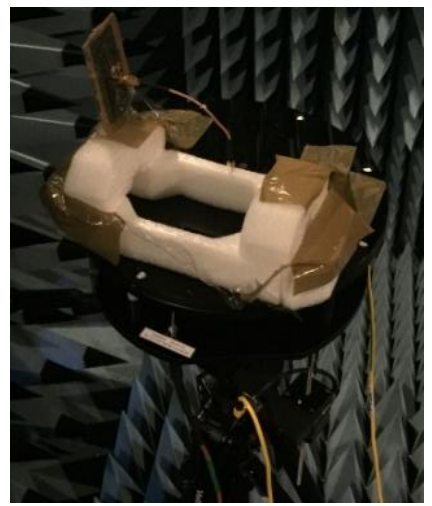

(b)

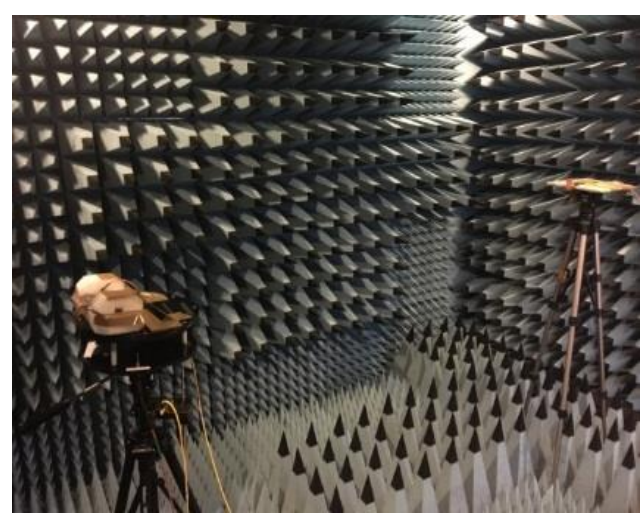

(c)

Figure 8. The antenna position of HTAB measured radiation pattern in (a) E-plane, (b) H-plane, (c) the environment of anechoic chamber. 
The result is transmitted to the Vector Network Analyser and then display in the antenna measurement studio software. The radiation pattern of the horn antenna to the standard dipole antenna is shown in figure 9; this is a referenced antenna used to calculate the gain of measured hooked antenna. The horn antenna has better match in frequency and the gain is $10 \mathrm{~dB}$. The measured pattern gain of horn antenna is gotten, the pattern maximum gain at $1.8 \mathrm{GHz}$ is $-42.92 \mathrm{~dB}$; at $2.1 \mathrm{GHz}$, the pattern maximum gain is $-44.38 \mathrm{~dB}$; and the pattern maximum gain is $-42.66 \mathrm{~dB}$ at $2.45 \mathrm{GHz}$.

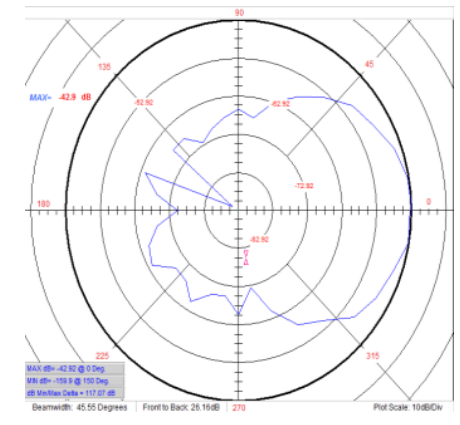

(a)

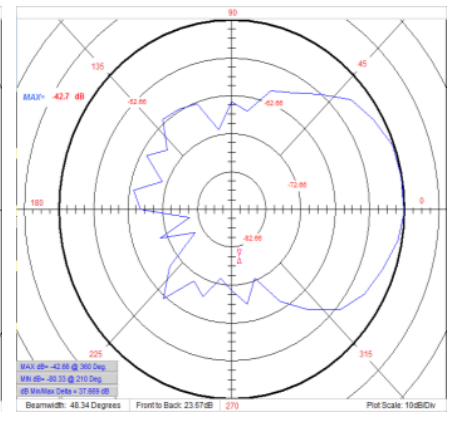

(b)

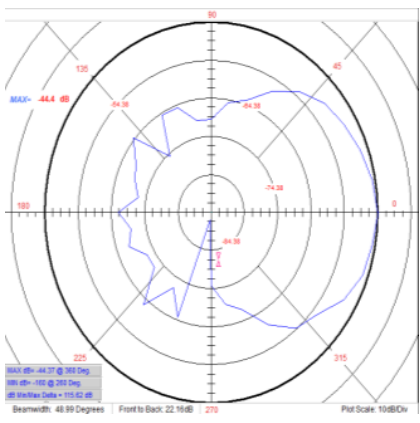

(c)

Figure 9. The radiation pattern E-plane of standard horn antenna at: (a) $f=1.8 \mathrm{GHz},(\mathbf{b}) \mathrm{f}=2.1 \mathrm{GHz}$, (c) $\mathrm{f}=2.45 \mathrm{GHz}$.

The E-plane of the wearable wideband hooked antenna at $1.8 \mathrm{GHz}, 2.1 \mathrm{GHz}$ and $2.45 \mathrm{GHz}$ is presented in figure 10, the patter maximum gain of them is $-52.8 \mathrm{~dB},-52.8 \mathrm{~dB}$ and $-54 \mathrm{~dB}$ separately. The shapes of three different frequencies are not good, but we can see that they look like a transverse number 8 shape as well.

Then we can calculate the antenna gain by this,

$$
\mathrm{G}=\text { Gmeasured }- \text { Ghorn }+10 \mathrm{~dB} \text {. }
$$

When $\mathrm{f}=1.8 \mathrm{GHz}$, the $\mathrm{G}_{1}=-52.8+42.92+10=0.12 \mathrm{~dB}$,

When $\mathrm{f}=2.1 \mathrm{GHz}$, the $\mathrm{G}_{2}=-52.8+44.38+10=1.58 \mathrm{~dB}$,

When $\mathrm{f}=2.45 \mathrm{GHz}$, the $\mathrm{G}_{3}=-54+42.66+10=-1.34 \mathrm{~dB}$.

And the simulated gain is $1.93 \mathrm{~dB}, 1.87 \mathrm{~dB}$ and $2.25 \mathrm{~dB}$ separately as shown before in figure 7 .

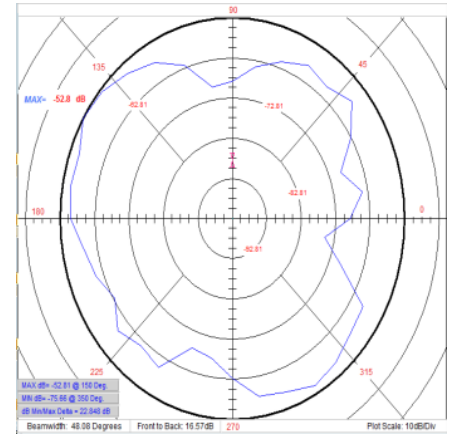

(a)

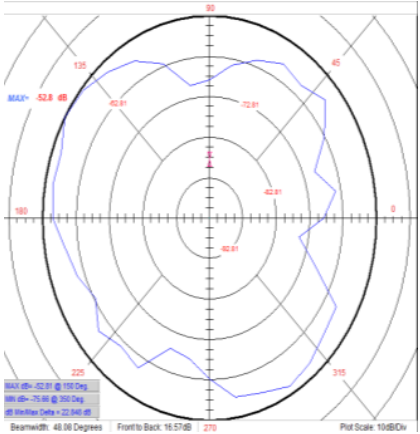

(b)

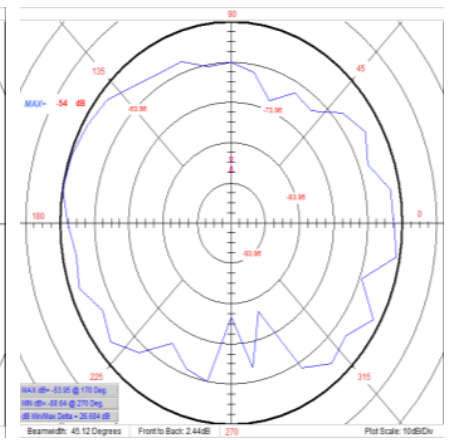

(c)

Figure 10. Measured E-plane of the HATB at: (a) $f=1.8 \mathrm{GHz},(\mathbf{b}) \mathrm{f}=2.1 \mathrm{GHz},(\mathbf{c}) \mathrm{f}=2.45 \mathrm{GHz}$.

In $1.8 \mathrm{GHz}$, it seems that the gain is little lower than the simulated result about $1.71 \mathrm{~dB}$. But in $2.1 \mathrm{GHz}$, the gain of measured is almost same as the simulated gain with only $0.29 \mathrm{~dB}$ difference. In $2.45 \mathrm{GHz}$, it is lower than the simulated gain with $3.6 \mathrm{~dB}$ difference. The reason why there are 
discrepancies between the measured and simulated results of antenna pattern and gain is mainly the frequency unmatching. The standard dipole antenna we used in pattern measurement has a narrow band. However the measured antenna is centered from $1.8 \mathrm{GHz}$ to $2.5 \mathrm{GHz}$, which is a wideband antenna. Hence, the power of measured antenna in this frequency band cannot received wholeheartedly by the dipole and vise versa. And there is a little path loss in the transmission. The Free Space Path Loss need to be considered in formula 7, it is known that the higer the frequency is, the path loss is more extensive as well. In $2.45 \mathrm{GHz}$, the Free Space Path Loss is $2.5 \mathrm{~dB}$ larger than 1.8 GHz.

$$
\text { FSPL }=20 \log _{10}(d)+20 \log _{10}(f)+20 \log _{10}\left(\frac{4 \pi}{c}\right)
$$

Afterward, to realize the transition from RF signal to the DC voltage, the rectifier PCC110 is utilized, which is called power management specialized power harvester RF - DC converter IC (PMIC). It has high conversion efficiency, which reaches up to $75 \%$ and enables long-range applications for converting low-level RF signals. Possessing wide frequency operating band which ranges from $100 \mathrm{MHz}$ to $6 \mathrm{GHz}$, harvesting from all modulation types. The PCC 110 has 3 pins; the left single end is the input end which is connected to the output end of antenna; in right side, the top one is the output end and bottom one is welded to the ground.

\subsection{The output voltage and efficiency measurement of rectifier}

Lastly, connecting the antenna with the rectifier input end by a coaxial line. Then using a multimeter to measure the output voltage from a $120 \mathrm{~K} \mathrm{Ohm} \mathrm{resisitor,} \mathrm{which} \mathrm{is} \mathrm{soldered} \mathrm{to} \mathrm{the} \mathrm{out}$ end of rectifier, as shown in figure 11. There is an outstanding signal and the output voltage is 0.056 volt, due to a perfectly matched and resonated dipole. Then the output power of rectifier can be gotten,

$$
P_{\text {out } 1}=\frac{V^{2}}{R}=\frac{0.056^{2} V^{2}}{120 \times 10^{3} \Omega}=26.1 \mathrm{nW}
$$

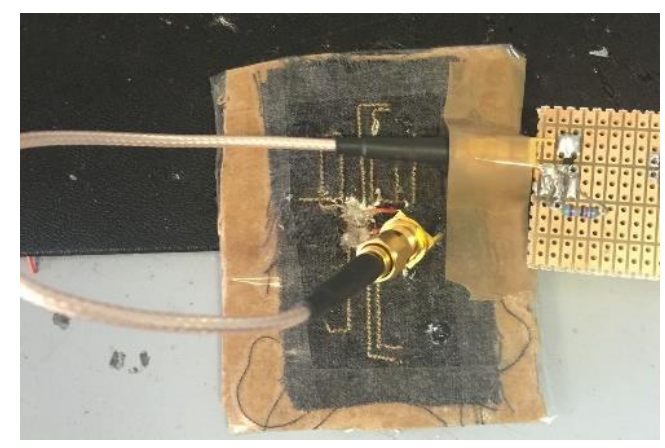

Figure 11. Proto of the wearable harvester with the rectifier board.

The output power of the antenna needs to be measured before calculating the efficiency of rectifier. We used the Spectrum Analyser to measure it under the ambient environment. After calibration, setting the SA configuration. Then connecting the antenna to this analyzer, we got the output power of antenna as shown in figure 12. The total power of antenna is:

$$
\begin{aligned}
& P_{\text {out } 2}=P_{1}+P_{2}+P_{3}+P_{4}=-44.5-51.17-60.17-57.5 \mathrm{dBm} \\
& =35.48 \mathrm{nW}+7.64 \mathrm{nW}+1 \mathrm{nw}+1.8 \mathrm{nW}=45.92 \mathrm{nW} .
\end{aligned}
$$


Then, the efficiency of the rectifier is:

$$
\eta=\frac{P_{\text {out } 1}}{P_{\text {out } 2}}=\frac{26.1 \mathrm{nW}}{45.92 \mathrm{nW}}=56.8 \%
$$

It is in agreement with the predicted results. It is approximate and reasonable, considering the $70 \%$ efficiency of rectifier and loss of line and circuit, meanwhile the unstable signal strength in the ambient environment. The simulated antenna total efficiency is about 0.97 at $1.8 \mathrm{GHz}, 0.88$ at $2.1 \mathrm{GHz}$ and 0.92 at $2.45 \mathrm{GHz}$. They are all in excellent efficiency due to a perfectly matched and resonated dipole. Considering above all factor, the working status of antenna and rectifier is functional.

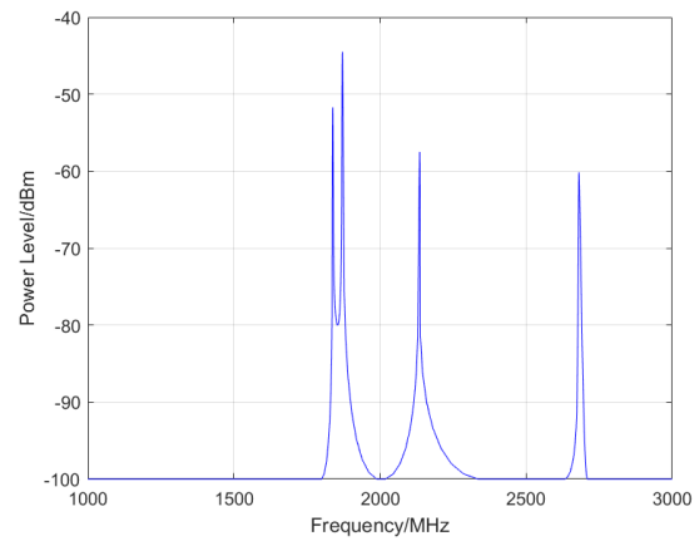

Figure 12. The waveform of the output power of wearable hooked antenna at different frequencies.

In the end, as the result shows, the voltage of this HATB is $0.056 \mathrm{~V}$ and the power is $26.1 \mathrm{nW}$ which could charge either the low power sensor devices or batteries. And if necessary, the antenna array could be used to enlarge the level of voltage or a voltage booster should be added to improve the input voltage and provide a regulated output voltage for a high-level load device. In addition, it is found from the results that when the wearable hooked antenna was placed on the body, compared to the return loss of alone antenna, each resonant frequencies has little shift which is about $30 \mathrm{MHz}$, but the bandwidth does not change a lot. This is caused by the change of substrate parameter from body interfere or the wrinkle of antenna on-body test. However, due to the wide frequency bandwidth, this change does not influence the performance of antenna much.

\section{Conclusion}

This paper presents a broadband wearable antenna fully weaved in textile and is integrated with a rectifier chip which is able to transfer RF signal to DC voltage and could operate with 2G, 3G, wifi, and 4G/5G frequency band simultaneously. The weaved antenna consists of three hooked shape antennas with tuning bars (HTAB) for impedance and bandwidth adjustment, which is designed after many other different tries. The simulated results of the HATB are nearly same as the measured results, except some special factors lead to the little changes in performance. Firstly, the measured antenna has operating bandwidth which ranges from $1.64 \mathrm{GHz}$ to $2.59 \mathrm{GHz}$ excepting the sector from $2.08 \mathrm{GHz}$ to $2.39 \mathrm{GHz}$ in $-10 \mathrm{~dB}$ reflection coefficient, while the simulated result has three sections of operating bandwidth, this is caused by the real fabrication difference, dielectri constant reduces due to the porous materials and the resonance point of these two dipoles are so close that the two closed 
waves were incorporated as one broadband $S_{11}$ wave, which is reasonable in the pratical making, it shows that the measured antenna has excellent performance in return loss. Moreover, as we know, the HATB has some frequency shift when on different substrates, especially on body, but the bandwidth has no much change, and this will not influence the work of the antenna, due to the wide operating band. Then, the dielectric of the fabric substrate has been measured and the conductive yarn is sewed in the substrate, which shows the sewed antennas are realizable and acceptable.

The radiation pattern of the sewed antenna in three different bands is nearly same as the simulated pattern in the gain, especially in $2.1 \mathrm{GHz}$. And the shape of pattern looks like a transverse number 8 shape which is same as the simulation one. After that, the gain of all frequencies are calculated, and as is foresighted that they are 1.71, 0.29 and $3.6 \mathrm{~dB}$ lower than the simulated gain of $1.93 \mathrm{~dB}, 1.87 \mathrm{~dB}$ and $2.25 \mathrm{~dB}$ separately. Afterward, to realize the transition from RF signal to DC voltage, the rectifier is utilized. In the end, there is a outstand output signal and the output voltage is about 0.056 volt, due to a perfectly matched and the resonated dipole. The measured output power of the antenna is summed, which is $45.92 \mathrm{nw}$. Then, the efficiency of rectifier is calculated, which is about $56.8 \%$, and it is in agreement with the predicted results.

Overall, the experimental results have indicated that making a multi-band wearable antenna with the textile yarn is possible. And it could be put into real use for wireless charging over long distance for IoT, IoV and other low power equipment. And the wearable antenna could be used for active RFID tag antenna as well with the power harvesting antenna for power supply to cover the needs of various environments and applications. The active RFID tag could be sweed in the rescureworkers suits easier for more comfortable and flexible move, and more efficient to accurately find the location of people without the environment and distance limitation. At the same time, more metal yarn can be used for fabricating the antenna array to harvest more energy. Then, it could be charged for the high-power charging devices and batteries. It is better than the other kinds of power charging system supporting all kinds of present mobile signal band including 5G signal which will be covered worldwide in the coming 5 years, and possessing with good efficiency, convenient fabricating, comfortable wearing and long-distance. With this harvesting architecture developement and popularization, the RF power in the air will be resued, then a green and promising networks will come ture in the future.

Author Contributions: conceptualization, W.H.Q. and Z.H.; methodology W.H.Q.; software, W.H.Q.; validation, W.H.Q. and Z.H.; formal analysis, W.H.Q.; investigation, W.H.Q. and L.X.; resources, W.H.Q. and L.X.; data curation, W.H.Q.; writing-original draft preparation, W.H.Q.; writing-review and editing, W.H.Q., S.M.W and Z.H.; visualization, W.H.Q.; supervision, Z.H.

Funding: This research received no external funding.

Acknowledgments: The author would to thank Yuxi Shi in School of Material of University of Manchester, helps and instructs me to sew it by myself. In addition, the portion of substrate material measurement is supported by Dr. Arthur Haigh and Zheng Li in electronic lab, University of Manchester. Thanks for the help of rectenna measurement from David Zhang, Kewen Pan, Graham and etc in school of EEE, University of Manchester. In the end, thank my supervisor Dr. Zhirun Hu who guides my whole project.

Conflicts of Interest: The authors declare no conflict of interest.

\section{References}

1. Shafique, K.; Khawaja, B. A.; Khurram, M. D.; Sibtain, S. M.; Yang, X. Energy harvesting using a low-cost rectenna for internet of things (iot) applications. IEEE Access, 2018, 99, 1-1. 
2. Deshmukh, S.D.; Shilaskar, S.N. Wearable sensors and patient monitoring system: A review. In Proceedings of the International Conference on Pervasive Computing, St-Louis, MI, USA, 23-27 March 2015.

3. Chen, Y. Challenges and opportunities of internet of things. Drayson Technologies 2015, 17th Asia and South Pacific Design Automation Conference, Jan. 30, 2012-Feb. 2 2012, pp.383-388.

4. Borza, P.N.; Machedon-Pisu, M.; Hamza-Lup, F. Design of Wireless Sensors for IoT with Energy Storage and Communication Channel Heterogeneity. Sensors 2019, 19, 3364.

5. Vyas, R.; Lakafosis, V.; Tentzeris, M.; Nishimoto, H.; Kawahara, Y. A battery-less, wireless mote for scavenging wireless power at UHF (470-570 MHz) frequencies, in IEEE AP-S Int. Symp./USNC/URSI Nat. Radio Sci. Meeting, Jul. 3-8, 2011, 10, pp. 69-72.

6. Liang, J.; Chiau, C. C.; Chen, X.; Parini, C. G. Study of a printed circular disc monopole antenna for UWB systems, IEEE Transactions on Antennas and Propagation, Nov. 2005, Vol-53, pp. 3500 - 3504.

7. Huang, H-F.; Zhang, S.-F. A compact triple-band monopole antenna for WLAN/WIMAX application, 2013 Proceedings of the International Symposium on Antennas \& Propagation (ISAP), vol. 01, 2013, pp. $454-457$.

8. Wong, K.; Hsu, W. A broad-band rectangular patch antenna with a pair of wide slits, IEEE Transactions on Antennas and Propagation, Sep 2001, Vol-49, pp. 1345 - 1347.

9. Mansour, M.; Le Polozec, X.; Kanaya, H. Enhanced Broadband RF Differential Rectifier Integrated with Archimedean Spiral Antenna for Wireless Energy Harvesting Applications. Sensors 2019, 19, 655.

10. Antonio A.A.; José M.F.G.; Pablo P.; Manuel S.C. Comparison Between a Multiband PIFA and a Ultrawideband Archimedean Spiral Antenna for Energy Harvesting in Microwave Bands. 2nd URSI ATRASC, Gran Canaria, 28 May - 1 June 2018.

11. Trinh-Van, S.; Lee, J.M.; Yang, Y.; Lee, K.-Y.; Hwang, K.C. Improvement of RF Wireless Power Transmission Using a Circularly Polarized Retrodirective Antenna Array with EBG Structures. Appl. Sci. 2018, 8, 324.

12. Hall, P.S.; Hao, Y. Antennas and Propagation for Body-Centric Wireless Networks, 2nd ed.; Artech House: Norwood, MA, USA, 2012.

13. Hall, P.S. Antennas challenges for body centric communications. In International Workshop on Antenna Technology (IWAT’07), Cambridge, UK, 21-23 March 2007; pp. 41-44

14. Salter, T.; Metze, G.; Goldsman, N. Parasitic aware optimization of an RF power scavenging circuit with applications to smart dust sensor networks, in IEEE Radio Wireless Symp., 2009; pp. 332-335.

15. Nintanavongsa, P.; Muncuk, U.; Lewis, D.R.; Chowdhury, K.R. Design optimization and implementation for RF energy harvesting circuits. IEEE J. Emerg. Sel. Top. Circuits Syst. 2012, 2, 24-33.

16. “SENSIMED Triggerfish ${ }^{\circledR}$-Continuous IOP monitoring," SENSIMED, Lausanne, Switzerland, Mar. 2012. Available online: http://www.sensimed.com/S-Trig-glaucoma.htm (Accessed on 9th May 2018).

17. Haerinia, M.; Noghanian, S. A Printed Wearable Dual-Band Antenna for Wireless Power Transfer. Sensors 2019, 19, 1732.

18. Roh, J.; Chi, Y. Embroidered Wearable Multiresonant Folded Dipole Antenna for FM Reception. IEEE Antennas \& Wireless Propagation Letter, 2010, Vol-9, pp. 803-806

19. Almohaimeed, A.M.; Amaya, R.E.; Lima, J.A.; Yagoub, M.C.E. An Adaptive Power Harvester with Active Load Modulation for Highly Efficient Short/Long Range RF WPT Applications. Electronics 2018, 7, 125.

20. Walaa, I.; Mahmoud A.; Abdelmegeed A.; Ahmed M.; Hesham E. A Compact and Dual Band Metamaterial Substrate Integrated Waveguide Antenna, IEEE AP-S International Antenna and Propagation Symposium Digest, Orlando, USA, Jun. 7-13, 2013, pp. 966-967.

21. Chahat, N.; Zhadobov, M.; Sauleau, R.; Mahdjoubi, K. Improvement of the On-Body Performance of a Dual-Band Textile Antenna using an EBG Structure. Antennas and Propagation Conference (LAPC), 2010 Loughborough. IEEE, 2010.

22. Zhu, S.; Langley R. Dual-band wearable Textile antennas on an EBG substrate. IEEE Transactions on Antennas and Propagation, April 2009, Vol. 57(4), pp. 926-935.

23. Boeykens, F.; Rogier, H.; Vallozzi, L. An efficient technique based on polynomial chaos to model the uncertainty in the resonance frequency of textile antennas due to bending. IEEE Trans. Antennas Propag. 2014, 62, 1253-1260.

24. Kennedy, T. F.; Fink, P. W.; Chu, A. W.; Champagne, N. J.; Lin, G. Y.; Khayat, M. A. Body-worn e-textile antennas: the good, the low-mass, and the conformal. IEEE Transactions on Antennas and Propagation, 2019, 57(4), 910-918. 
25. Salvado, R.; Loss, C.; Gonçalves, R.; Pinho, P. Textile Materials for the Design of Wearable Antennas: A Survey. Sensors 2012, 12, 15841-15857.

26. Wang, X.; Abdelatty, O.; Mortazawi, A. Design of a wide dynamic range rectifier array with an adaptive power distribution technique. In Proceedings of the 2016 46th European Microwave Conference (EuMC), London, UK, 4-6 October 2016; pp. 922-925.

27. Paing, T.; Falkenstein, E. A.; Zane, R.; Popovic, Z. Custom IC for ultralow power RF energy scavenging. IEEE Transactions on Power Electronics, 2011, 26(6), 1620-1626.

28. Musil, J.; Zacek, F. Microwave Measurements of Complex Permittivity by Free Space Methods, Elsevier, Amsterdam, 1986.

29. Haigh, A.D.; Thompson, F.; Gibson, A.A. P. Complex Permittivity of Liquid and Granular Materials using Waveguide Cells, October 2001, 2, 425-434.

30. Ball, J.A.R.; Horsfield, B. Resolving ambiguity in broadband waveguide permittivity measurement on moist materials. IEEE Trans. Instrum. Meas. 1998, 47, 390-392.

31. Baker-Jarvis, J.; Janezic, M.D.; DeGroot, D.C. High-Frequency Dielectric Measurements. IEEE Trans. Instrum. Meas. 2010, 13, 24-31. 